\title{
Evening primrose oil in rheumatoid arthritis: changes in serum lipids and fatty acids
}

\author{
J JÄNTTI, ${ }^{1}$ T NIKKARI, ${ }^{2}$ T SOLAKIVI ${ }^{2}$ H VAPAATALO,${ }^{2}$ \\ AND H ISOMÄK I
}

From the ${ }^{1}$ Rheumatism Foundation Hospital, Heinola, Finland, and the ${ }^{2}$ Department of Biomedical Sciences, University of Tampere, Tampere, Finland

SUMMARY The serum concentration of lipids and composition of fatty acids after overnight $\dot{\infty}_{\infty}^{+}$ fasting were studied in 18 patients with rheumatoid arthritis treated for 12 weeks with either 20 mliv of evening primrose oil containing $9 \%$ of $\gamma$-linolenic acid or olive oil. The serum concentrations $\vec{N}$ of oleic acid, eicosapentaenoic acid, and apolipoprotein B decreased and those of linoleic acid,,$\circ$ $\gamma$-linolenic acid, dihomo- $\gamma$-linolenic acid, and arachidonic acid increased during treatment with ${ }_{-}^{\supset}$ evening primrose oil. During olive oil treatment the serum concentration of eicosapentaenoic acid decreased and those of high density lipoprotein-cholesterol and apolipoprotein A-IO increased slightly. The decrease in serum eicosapentaenoic acid and the increase in arachidonicon acid concentrations induced by evening primrose oil may not be favourable effects in patients with rheumatoid arthritis in the light of the roles of these fatty acids as precursors of eicosanoidg.

Evening primrose oil (EPO) is currently one of the most popular unorthodox remedies for rheumatoid arthritis (RA). The rationale of its therapeutic use is based on the high amount $(9 \%)$ of $\gamma$-linolenic acid, which is metabolised into dihomo- $\gamma$-linolenic acid, the natural precursor of the 1-series prostaglandins containing one double bond. Prostaglandins of the 1-series have been shown to possess antiinflammatory activity in experimental animal models. ${ }^{1}$ By replacing arachidonic acid, precursor of the proinflammatory prostaglandins of the 2-series, as a substrate for cyclo-oxygenase, dietary $\gamma$ linolenic acid has been thought to have a beneficial effect on the prostaglandin synthesis in RA.

Controlled studies on EPO in man have been carried out in atopic eczema, ${ }^{2}$ in Sjögren's syndrome, ${ }^{3}$ and in RA. ${ }^{4}$ No clinical effect or change in plasma or urinary prostaglandin $E_{1}$, cAMP, or cGMP concentrations was noted during 12 weeks of treatment with EPO ${ }^{4}$ The recent report of Belch et al showed no improvement in the objective markers of inflammation, but the subjective response of the patients to EPO was better than that to olive oil. ${ }^{5}$ In another study olive oil significantly improved the symptoms of RA. ${ }^{6}$

Accepted for publication 14 June 1988 .

Correspondence to Dr J Jäntti, Rheumatism Foundation Hospital, SF-18120 Heinola, Finland.
We carried out a double blind study in 20 patients with RA, using EPO and olive oil. No differences were noted between the two treatment groups in $\frac{0}{D}$ clinical signs, laboratory indicators of inflammatory $\cong$ activity, or plasma prostanoid concentrations (Jänttio $\overrightarrow{\overrightarrow{0}}$ $\mathrm{J}$, unpublished results). We now report changes in 3 the serum lipids of these patients treated for 12 ? weeks with either EPO or olive oil.

\section{Patients and methods}

Twenty patients with definite or classical RA, willing to participate in the study, and accepting the 13 weeks minimum of absolute abstinence from non-o steroidal anti-inflammatory drugs (NSAIDs), were? randomly allocated into two treatment groups of 10 음 patients each. The treatment was either olive oil 10 ml twice daily or evening primrose oil $10 \mathrm{ml}$ twice daily. The oils were packed in numbered identical $N$ bottles, and the code was not broken until all the N analyses had been carried out. The oils were ${ }_{\sigma}^{\omega}$ supplied by Bio-Oil Research Ltd, Crewe, England. Table 1 shows the fatty acid compositions of the oils, determined as described below.

Before the start of the study all patients abstained from NSAIDs for seven days and in the case ofo aspirin for 10 days. No NSAIDs were allowed during the study. Paracetamol was allowed as a pain killer, if necessary. Long term antirheumatic drug 
Table 1 Fatty acid compositions of the oils. Values are percentages of total fatty acids

\begin{tabular}{lcc}
\hline Fatty acid & $E P O^{*}$ & Olive oil \\
\hline Palmitic & $5 \cdot 7$ & $9 \cdot 8$ \\
Stearic & $1 \cdot 6$ & $3 \cdot 3$ \\
Oleic & $11 \cdot 4$ & $78 \cdot 1$ \\
Linoleic & $71 \cdot 3$ & $6 \cdot 3$ \\
-Linolenic & $0 \cdot 3$ & $0 \cdot 7$ \\
$\gamma$-Linolenic & $8 \cdot 6$ & $0 \cdot 0$ \\
\hline
\end{tabular}

${ }^{*} \mathrm{EPO}=$ evening primrose oil.

treatment was continued throughout the study on an unchanged dosage. In the EPO group five patients received intramuscular gold and two patients hydroxychloroquine. In the olive oil group four patients received intramuscular gold, three patients hydroxychloroquine, one patient penicillamine, and one patient prednisolone $5 \mathrm{mg} /$ day.

The mean age was 50 years in the EPO group and 38 years in the olive oil group $(p<0 \cdot 05)$. The duration of RA was 13 years in the EPO group and 10 years in the olive oil group $(p>0.05)$. Nine patients in the EPO group and eight in the olive oil group had positive rheumatoid factor. Both groups comprised one man and nine women. One patient in both groups discontinued the study.

The serum samples for fatty acid and apolipoprotein analysis were taken after overnight fasting before the treatment and six and 12 weeks later. The serum samples were stored at $-20^{\circ} \mathrm{C}$ and analysed simultaneously. Apolipoproteins A-I and B were studied only in three patients with EPO and in four patients with olive oil treatment. Serum total cholesterol, high density lipoprotein (HDL)cholesterol, and triglycerides were studied according to the hospital routine on the same day as the sample was taken.

For fatty acid analysis serum lipids were extracted with chloroform-methanol (1:1) and saponified with $\mathrm{KOH}$-ethanol. The fatty acids were methylated with $\mathrm{HCl}$-methanol and analysed in a Hewlett-Packard $5880 \mathrm{~A}$ gas chromatograph using an open tubular quartz capillary column of OV-351 (Nordion Instruments, Ltd, Finland) and a splitless injection technique. The temperature was programmed from $100^{\circ}$ to $240^{\circ} \mathrm{C}$. Peaks generated by a flame ionisation detector were measured with a Hewlett-Packard electronic integrator. The results were expressed as percentages of the total area of all peaks from 14:0 to $22: 6$.

\section{Results}

Tables 2 and 3 show the mean values (SD) of serum cholesterol, HDL-cholesterol, triglycerides, apolipoproteins A-I and B, and fatty acids in the two treatment groups. As expected, EPO significantly increased the proportion of linoleic acid, $\gamma$-linolenic acid, and dihomo- $\gamma$-linolenic acid, whereas olive oil had no effect on these fatty acids. EPO also increased the percentage of arachidonic acid, though the rise reached only marginal significance after six weeks. The main displaced fatty acid in the EPO treated group was oleic acid, but EPO also highly significantly decreased the percentage of eicosapentaenoic acid after six weeks, and still more after 12 weeks. Olive oil also decreased eicosapentaenoic acid after 12 weeks. The percentages of

Table 2 Effects of evening primrose oil (20 ml/day) on the serum lipids in nine patients with RA. Values are mean (SD)

\begin{tabular}{|c|c|c|c|}
\hline Lipid & Before treatment & 6 Weeks later & 12 Weeks later \\
\hline \multicolumn{4}{|l|}{ Fatty acids: } \\
\hline Oleic $(\%)$ & $25 \cdot 2(2 \cdot 7)$ & $19.9(2 \cdot 2)^{* * *}$ & $19 \cdot 0(1.6)^{* * *}$ \\
\hline Linoleic (\%) & $25 \cdot 8(4 \cdot 3)$ & $31 \cdot 3(4 \cdot 7)^{* * *}$ & $32 \cdot 1(4 \cdot 9)^{* * *}$ \\
\hline$\gamma$-Linolenic (\%) & $0.4(0 \cdot 1)$ & $1 \cdot 1(0 \cdot 2)^{* * *}$ & $1 \cdot 1(0 \cdot 3)^{* * *}$ \\
\hline Dihomo- $\gamma$-linolenic $(\%)$ & $1.5(0 \cdot 2)$ & $2 \cdot 7(0 \cdot 7)^{* * *}$ & $3.0(0.9)^{* * *}$ \\
\hline Arachidonic (\%) & $5 \cdot 3(1 \cdot 4)$ & $6 \cdot 5(1 \cdot 3)^{*}$ & $6.4(1.6)$ \\
\hline$\alpha$-Linolenic (\%) & $0.7(0.2)$ & $0.5(0 \cdot 1)$ & $0 \cdot 6(0 \cdot 2)$ \\
\hline Eicosapentaenoic (\%) & $1.2(0 \cdot 5)$ & $0 \cdot 8(0 \cdot 3)^{* * *}$ & $0 \cdot 7(0 \cdot 3)^{* * *}$ \\
\hline Total cholesterol $(\mathrm{mmol} / \mathrm{l})$ & $5 \cdot 5(1 \cdot 8)$ & $5 \cdot 2(1 \cdot 4)$ & $5 \cdot 3(1 \cdot 3)$ \\
\hline HDLt-cholesterol $(\mathrm{mmol} / \mathrm{l})$ & $1.5(0 \cdot 3)$ & $1.6(0 \cdot 6)$ & $1.6(0.5)$ \\
\hline Triglycerides (mmoll $)$ & $1 \cdot 2(0 \cdot 4)$ & $1 \cdot 1(0 \cdot 5)$ & $1 \cdot 2(0 \cdot 4)$ \\
\hline Apolipoprotein A-I $(\mathrm{g} / \mathrm{l}) \ddagger$ & $1 \cdot 366(0 \cdot 158)$ & $1 \cdot 374(0 \cdot 204)$ & $1 \cdot 330(0 \cdot 197)$ \\
\hline Apolipoprotein B $(\mathrm{g} / \mathrm{l}) \ddagger$ & $1.445(0 \cdot 237)$ & $1.228(0.193)^{*}$ & $1 \cdot 149(0 \cdot 259)^{* *}$ \\
\hline
\end{tabular}

Significance of the change during treatment $\left(t\right.$ test for paired values): ${ }^{*} \mathrm{p}<0 \cdot 05 ;{ }^{* *} \mathrm{p}<0 \cdot 01 ;{ }^{* * *} \mathrm{p}<0 \cdot 001$. $\dagger \mathrm{HDL}=$ high density lipoprotein.

$\ddagger$ Three patients. 
Table 3 Effects of olive oil (20 ml/day) on the serum lipids in nine patients with RA. Values are mean (SD)

\begin{tabular}{|c|c|c|c|}
\hline Lipid & Before treatment & 6 Weeks later & 12 Weeks later \\
\hline \multicolumn{4}{|l|}{ Fatty acids: } \\
\hline Oleic (\%) & $24.4(3.0)$ & $25 \cdot 2(3 \cdot 3)$ & $25 \cdot 8(3 \cdot 9)$ \\
\hline Linoleic (\%) & $29 \cdot 8(5 \cdot 3)$ & $31 \cdot 7(4 \cdot 8)$ & $31 \cdot 8(5 \cdot 4)$ \\
\hline$\gamma$-Linolenic $(\%)$ & $0.2(0.9)$ & $0.3(0.2)$ & $0.3(0 \cdot 2)$ \\
\hline Dihomo- $\gamma$-linolenic (\%) & $1 \cdot 2(0 \cdot 3)$ & $1 \cdot 3(0 \cdot 3)$ & $1 \cdot 3(0 \cdot 2)$ \\
\hline Arachidonic (\%) & $4.7(0.7)$ & $4 \cdot 7(0 \cdot 8)$ & $4 \cdot 3(0 \cdot 7)$ \\
\hline$\alpha$-Linolenic (\%) & $0.7(0 \cdot 2)$ & $0.7(0.2)$ & $0.7(0.2)$ \\
\hline Eicosapentaenoic (\%) & $1.4(1 \cdot 0)$ & $1 \cdot 0(0 \cdot 3)$ & $0.9(0 \cdot 3)^{*}$ \\
\hline Total cholesterol (mmol/l) & $5 \cdot 0(1 \cdot 2)$ & $5 \cdot 2(1 \cdot 2)$ & $5 \cdot 2(1 \cdot 3)$ \\
\hline HDL†-cholesterol (mmol/l) & $1 \cdot 2(0 \cdot 3)$ & $1.4(0.5)$ & $1.5(0.7)^{*}$ \\
\hline Triglycerides (mmol/l) & $1 \cdot 2(0 \cdot 3)$ & $1 \cdot 0(0 \cdot 6)$ & $1 \cdot 2(0 \cdot 9)$ \\
\hline Apolipoprotein A-I (g/l) $\ddagger$ & $0.965(0.089)$ & $1 \cdot 127(0 \cdot 146)^{*}$ & $1 \cdot 188(0 \cdot 08)^{* * *}$ \\
\hline Apolipoprotein B $(\mathrm{g} / \mathrm{l}) \ddagger$ & $0.89(0.227)$ & $0.782(0 \cdot 156)$ & $0 \cdot 752(0 \cdot 133)$ \\
\hline
\end{tabular}

Significance of the change during treatment ( $t$ test for paired values): ${ }^{*} p<0 \cdot 05 ;{ }^{* * *} p<0 \cdot 001$.

†HDL=high density lipoprotein.

$\ddagger$ Four patients.

the serum fatty acids did not differ significantly between the two groups before treatment. After 12 weeks the oleic acid level was lower $(p<0.001)$, the $\gamma$-linolenic and dihomo- $\gamma$-linolenic acid values were higher $(\mathrm{p}<0 \cdot 001)$, and the arachidonic acid content was higher $(p<0.01)$ in patients treated with EPO than in patients treated with olive oil ( $t$ test for unpaired values).

Serum total cholesterol and triglyceride concentrations did not change in either of the groups, but the serum HDL-cholesterol concentration increased slightly during olive oil treatment. EPO had no effect on the serum concentration of apolipoprotein A-I, whereas that of apolipoprotein B decreased in all three patients studied. Apolipoprotein A-I increased in all four studied patients treated with olive oil, whereas apolipoprotein B decreased in three patients.

\section{Discussion}

EPO increased the proportion of $\gamma$-linolenic and dihomo- $\gamma$-linolenic acids in serum fatty acids as expected, but it also increased the proportion of arachidonic acid and decreased that of eicosapentaenoic acid. This is in accordance with earlier results, ${ }^{7}$ and is probably due to competition with the high proportion of $\omega 6$ fatty acids, linoleate and $\gamma$ linoleate in EPO for the enzymes metabolising polyunsaturated fatty acids, including eicosapentaenoic acid.

As $\gamma$-linolenic and dihomo- $\gamma$-linolenic acids are precursors of the 1-series prostaglandins, which have anti-inflammatory properties, the observed increase of these fatty acids in serum lipids can be regarded as a favourable effect. On the other hand, as arachidonic acid is the precursor of proinflammatory prostaglandins of the 2-series and leucotrienes $\overrightarrow{0}$ of the 4-series its observed increase in serum lipig\& as a result of EPO treatment could potentially be harmful. Raised arachidonic acid levels may expla望 the higher concentrations of thromboxane $B_{2} \overrightarrow{\text { in }}$ five out of nine patients with RA treated with EPO, though the prostaglandin $\mathrm{E}_{2}$ concentrations in plasma showed no significant changes (Jäntti $J$, unpublished results).

Eicosapentaenoic acid is a precursor of the biologically less active prostaglandins of the 3-series. It may even possess anti-inflammatory effects by inhibiting the 5-lipoxygenase pathway in neutrophils and monocytes and by antagonising the leucotriene $B_{4}$ mediated functions of neutrophils. ${ }^{8}$ Treatment of RA with eicosapentaenoic acid has given promising though not excellent results. ${ }^{9} 10$ In addition, this acid has several favourable cardiovascular effects in man. ${ }^{11}$ Thus the observed decrease in the proportion of eicosapentaenoic acid in the plasma fatty acids $\frac{\text { T }}{2}$ can be regarded as unfavourable. Whether these theoretical suggestions are clinically relevant is not $N$ known, but they make EPO a questionable treat- $N$ ment for RA.

The total serum cholesterol concentrations in $\omega$ patients with active RA are lower than those of 2 healthy people. In patients treated with slow acting $\stackrel{\circ}{\subseteq}$ antirheumatic drugs the low serum cholesterol values increase towards normal, whereas the ${ }^{+}$ NSAIDs have no effect. ${ }^{12}$ Most of our patients $\frac{T}{\circ}$ received slow acting antirheumatic drugs, but still $\stackrel{\odot}{\oplus}$ their serum cholesterol concentrations were rather $\frac{?}{\mathbb{Q}}$ low. The treatments did not influence the total 2 
cholesterol, though olive oil administration slightly increased the HDL-cholesterol and apolipoprotein A-I concentrations in serum. It has been shown earlier that polyunsaturated fatty acids in diet decrease both the total and the HDL-cholesterol concentrations, whereas oleic acid decreases only the total cholesterol value. ${ }^{13}$ As HDL-cholesterol and apolipoprotein A-I are negative risk factors for coronary heart disease olive oil appears to have more favourable effect than EPO in regard to this disease.

It can be concluded that during treatment of RA for 12 weeks with evening primrose oil significant changes took place in the serum fatty acids. Some of these changes are theoretically undesirable in the light of the role of these fatty acids as precursors of prostaglandins and leucotrienes.

\section{References}

1 Kunkel S L, Ogawa H, Ward P A, Zurier R B. Suppression of chronic inflammation by evening primrose oil. Prog Lipid Res 1982; 20: 885-8.

2 Wright S, Burton J L. Oral evening-primrose-seed oil improves atopic eczema. Lancet 1982; ii: 1120-2.

3 Oxholm P, Manthorpe R, Prause J U, Horrobin D. Patients with primary Sjögren's syndrome treated for two months with evening primrose oil. Scand J Rheumatol 1986; 15: 103-8.
4 Mørk Hansen T, Lerche A, Kassis V, Lorenzen I. Søndergaard J. Treatment of rheumatoid arthritis with prostaglandin $E_{1}$ precursors cis-linoleic acid and $\gamma$-linolenic acid. Scand $J$ Rheumatol 1983; 12: 85-8.

5 Belch J J F, Ansell D, Madhok R, O'Dowd A, Sturrock R D. Effects of altering dietary essential fatty acids on requirements for non-steroidal anti-inflammatory drugs in patients with rheumatoid arthritis: a double blind placebo controlled study, Ann Rheum Dis 1988; 47: 96-104.

6 Darlington L G, Ramsay N W. Olive oil for rheumatoid patients? Br J Rheumatol 1987 26: (suppl 2): 215.

7 Nikkari T. Serum fatty acids and coronary heart disease in Finnish populations. Prog Lipid Res 1986; 25: 437-50.

8 Lee T H, Hoover R L, Williams J D, et al. Effect of dietary enrichment with eicosapentaenoic and docosahexaenoic acids on in vitro neutrophil and monocyte leukotriene generation and neutrophil function. $N$ Engl J Med 1985; 312: 1217-24.

9 Kremer J M, Jubiz W, Michalek A, et al. Fish-oil fatty acid supplementation in active rheumatoid arthritis. A doubleblinded, controlled, crossover study. Ann Intern Med 1987; 106: 497-503.

10 Darlington L G. Do diets rich in polyunsaturated fatty acids affect disease activity in rheumatoid arthritis? Ann Rheum Dis 1988; 47: 169-72.

11 Leaf A, Weber P C. Cardiovascular effects of $n-3$ fatty acids. $N$ Engl J Med 1988; 318: 549-57.

12 Svensson K L G, Lithell H, Hällgren R, Selinus I, Vessby B. Serum lipoprotein in active rheumatoid arthritis and other chronic inflammatory arthritides. Arch Intern Med 1987; 147: 1912-20.

13 Grundy S M. Monounsaturated fatty acids, plasma cholesterol, and coronary heart disease. Am J Clin Nutr 1987; 45: 1168-75. 